\title{
Effect of $\beta$-carotene on cancer cell stemness and differentiation in SK-N-BE(2)C neuroblastoma cells
}

\author{
HYUN AH LEE, SEOLHYUN PARK and YURI KIM \\ Department of Nutritional Science and Food Management, Ewha Womans University, \\ Seodaemun-gu, Seoul 120-750, Republic of Korea
}

Received April 29, 2013; Accepted July 1, 2013

DOI: $10.3892 /$ or.2013.2643

\begin{abstract}
Neuroblastoma is a solid tumor often diagnosed in childhood. While there have been intense efforts to develop a treatment for neuroblastoma, current therapies remain unsuccessful due to high rate of resistance and metastasis. Most cancers originate from a subset of self-renewing cells, primarily cancer stem cells (CSCs), which establish a tumor through continuous self-renewal and differentiation. The successful elimination of CSCs is an important goal in the development of effective strategies to achieve complete remission for cancers. Although $\beta$-carotene has been associated with several anticancer mechanisms, the efficacy of $\beta$-carotene against CSCs remains unclear. In the present study, $\beta$-carotene was shown to reduce cell growth and induce neuronal cell differentiation, concomitant with a marked increase in the phosphorylation of extracellular signal-regulated kinases (ERK) (p42/p44). More importantly, $\beta$-carotene inhibited self-renewal characteristics of CSCs and decreased expression of several stem cell markers. Levels of mRNA and protein of Drosophila delta-like 1 homolog (Drosophila) (DLK1) were downregulated following treatment with $\beta$-carotene. In addition, knockdown of $D L K 1$ by siRNA enhanced the inhibitory effect of $\beta$-carotene on colony formation of neuroblastoma cells. $\beta$-carotene also potentiated the effect of cisplatin on the self-renewal characteristics of CSCs in neuroblastoma, revealing that $\beta$-carotene has the capacity to resensitize cells to cisplatin cytotoxicity by directly targeting CSCs. In conclusion, $\beta$-carotene was shown to strongly increase the anticancer efficacy against neuroblastoma cancer stem-like cells. Moreover, these results suggest that the targeting of CSCs is a novel mechanism of $\beta$-carotene. Thus, $\beta$-carotene is a potential chemotherapeutic reagent for this cancer.
\end{abstract}

Correspondence to: Dr Yuri Kim, Department of Nutritional Science and Food Management, Ewha Womans University, 52 Ewhayeodae-gil, Seodaemun-gu, Seoul 120-750, Republic of Korea E-mail: yuri.kim@ewha.ac.kr

Key words: $\beta$-carotene, neuroblastoma, cancer stem cells, deltalike 1 homolog

\section{Introduction}

Neuroblastoma (NB) is the most common solid tumor diagnosed in children, and it originates from immature neuronal cells of the sympathetic nervous system. NBs are also comprised of multiple cell phenotypes, including neuroblasts, non-neuronal cells and melanocytes (1). In NB cell lines, as well as in primary NB tumors, three distinct cell types have been identified: Schwannian/melanoblastic precursors (S-type), neuroblastic/neuroendocrine precursors (N-type), and morphologically intermediate stem cells (I-type). I-type stem cells represent precursors of both $\mathrm{N}$ - and S-type cells, and also represent the most immature and highly malignant population of NB cells (2). Based on significant similarities in the gene expression profiles of normal neuroblasts and malignant $\mathrm{NB}$, as well as the morphological and functional differentiation that can be achieved with NB cells in culture, the differentiation process of NB can be studied in vitro.

A growing body of evidence has demonstrated that many types of cancers are driven by a small population of cancer cells, termed cancer stem cells (CSCs), or cancer-initiation cells (3). CSCs have the capacity to undergo differentiation, self-renewal and tumor generation. Moreover, CSCs have the potential to induce tumor growth, metastasis and resistance to radiation and chemotherapy (4). Understanding the signaling pathways that mediate functional characteristics is important in preclinical models, including animal models or in vitro cell lines. Drosophila delta-like 1 homologue (DLK1) is a member of the epidermal growth factor-like homeotic protein family. DLK1 has been known to modulate differentiation signaling in adipocytes and hematopoietic stem cells and has been characterized as a bona fide stem cell gene in neuronal tumors $(5,6)$. Mitogen activated protein kinase (MAPK) signaling pathways have been shown to play pivotal roles in growth, differentiation and stress responses $(7,8)$. It has been reported that MAPK signaling is affected by the expression of DLK1 (9). Correspondingly, extracellular signal-regulated kinases $(E R K)(p 42 / 44)$ are phosphorylated during neuronal differentiation and the activation of MEK/ERK signaling was found to decrease the self-renewal capacity of embryonal stem cells $(8,10)$.

During tumor treatment, most cancer cells, including a majority of differentiated tumor cells and actively replicating cells, respond to cytotoxic treatments. However, acquired 
multidrug resistance to conventional therapies can develop in CSCs. Therefore, the successful elimination of both actively replicating tumor cells, and slowly dividing CSCs, may represent a more effective strategy for the complete treatment of various types of cancer.

$\beta$-carotene (BC) is a provitamin A carotenoid, and a source of retinoids in dark green and orange fruits and vegetables. Observational studies have reported that an increase in the intake of dietary BC with increased BC plasma concentrations is associated with a decreased risk of several types of cancers $(11,12)$. Accumulating evidence also suggests that mechanisms involving antioxidant potential, alterations in intracellular signaling pathways, and regulation of cell proliferation and apoptosis, contribute to the anticarcinogenic effects associated with BC $(13,14)$. Retinoic acid (RA) has also been shown to promote the differentiation of embryonic stem cells and CSCs (15), and is used as a chemotherapeutic drug for various types of cancers (16). Moreover, direct, or indirect effects of several dietary compounds, including sulforaphane and curcumin on self-renewal pathways, have been identified $(17,18)$.

Currently, little is known about the role of $\mathrm{BC}$ in the differentiation and self-renewal characteristics of CSCs. Therefore, in the present study, the chemopreventive effects of BC on the induction of differentiation and attenuation of cancer cell stemness were investigated. Moreover, based on the potential for CSCs to contribute to chemoresistance, the chemotherapeutic effect of administering BC and cisplatin in combination was investigated, as well as the hypothesis that the effects of $\mathrm{BC}$ are mediated through the targeting of NB cancer stem celllike cells.

\section{Materials and methods}

Reagents and cell culture. BC, all-trans-retinoic acid (RA), tetrahydrofuran (THF) and cis-diamminedichloroplatinum(II) (cisplatin) were purchased from Sigma Chemical Co. (St. Louis, MO, USA). U0126 and PD98059 were obtained from Cell Signaling Technology Inc. (Danvers, MA, USA). Two human NB cell lines, SK-N-BE(2)C [BE(2)C] and SH-SY5Y (SY5Y), were obtained from the American Type Culture Collection (ATCC, Rockville, MD, USA) and maintained in a 1:1 mixture of Minimum Essential Medium (MEM) and Ham's F-12 medium supplemented with $10 \%$ fetal bovine serum (FBS) and antibiotics. BC was freshly prepared in THF containing $0.01 \%$ butylated hyroxytoluene (BHT), then added to the culture medium to a final concentration of 5-20 $\mu \mathrm{M}$. The concentration of THF in culture medium was adjusted to be the same in all experiments, and the final concentration did not exceed $0.4 \%(\mathrm{v} / \mathrm{v})$. The various concentrations of BC $(5,10$ and $20 \mu \mathrm{M}$ ) used in the present study were not associated with any significant cytotoxicity, and cell culture involving BC was performed under minimal light to minimize the breakdown of BC. Cisplatin was dissolved in $0.9 \% \mathrm{NaCl}$.

Cell viability assay. Cell viability was evaluated using 3-(4,5-dimethylthiazol-2-yl)-2,5-diphenyltetrazolium bromide (MTT; Sigma) (19). Briefly, cells were seeded in 96-well plates and incubated overnight at $37^{\circ} \mathrm{C}$ with $5 \% \mathrm{CO}_{2}$. Varying concentrations of $\mathrm{BC}$ were added to the wells and incubated for 48-96 h. MTT was then added to each well at a final concentration of $500 \mu \mathrm{g} / \mathrm{ml}$. After $4 \mathrm{~h}$ at $37^{\circ} \mathrm{C}$, the supernatant from each well was removed. Cells were lysed with DMSO, and the absorbance values at $560 \mathrm{~nm}$ were measured using a microplate reader (Molecular Devices, Sunnyvale, CA, USA).

Measurement of neurite outgrowth. $\mathrm{BE}(2) \mathrm{C}$ cells were plated in triplicate in 6 -well plates $\left(4 \times 10^{4}\right.$ cells/well) and treated with varying doses of $\mathrm{BC}$ for 5 days. A process having at least twice the diameter of the associated cell body, and possessing a terminal growth edge, was considered as a neurite. The percentage of cells with outgrowing neurites in $\sim 50$ cells/per well was counted. The mean and standard error of the mean (SEM) are reported. Three independent experiments for each treatment were performed.

Clonogenic assays. To determine the self-renewal characteristics of cells, clonogenic assays were performed as previously described (20). Briefly, BE(2)C cells were plated in 6-well plates (500 cells/well) and treated with varying concentrations of BC for 10 days. After fixing the cells with $0.9 \% \mathrm{NaCl}$, colonies were stained with crystal violet (Sigma). Colonies containing at least 50 stained cells were counted. Plating efficiency (PE) was calculated as follows: $\mathrm{PE}=($ colony number/total cell number) $\mathrm{x} 100 \%$.

Sphere formation assays. To facilitate sphere formation, poly-(2-hydroxyethyl methacrylate) (polyHEMA; SigmaAldrich)-coated plates were prepared by applying $500 \mu \mathrm{l}$ of $10 \%$ polyHEMA stock solution [12\% polyHEMA solution in $95 \%$ ethanol $(\mathrm{w} / \mathrm{v})]$ into each well of a 6 -well plate $(21)$. Sphere medium was prepared with DMEM-F12 (3:1; Invitrogen, Carlsbad, CA, USA) containing 2\% B27 (Invitrogen), $20 \mathrm{ng} / \mathrm{ml}$ recombinant human epidermal growth factor and $40 \mathrm{ng} / \mathrm{ml}$ recombinant human fibroblast growth factor basic (PeproTech, Rocky Hill, NJ, USA). After 6 days, the number of tumor spheres containing $>50$ cells was counted.

Antibodies and western blot assay. Western blot assays were performed as previously described (22). Briefly, cell lysates were separated by SDS-PAGE and transferred onto polyvinylidene fluoride (PVDF) membranes (Millipore, Billerica, MA, USA). The membranes were incubated with $5 \%$ non-fat dried milk, followed by antibodies against DLK1, SOX2 (Millipore), Notch1 (Novus Biologicals, Littleton, CO, USA), $\beta$-tubulin III (Sigma), phospho-ERK (p42/p44), total ERK (Cell Signaling), or $\beta$-actin (Santa Cruz Biotechnology, Santa Cruz, CA, USA). Bound antibodies were detected using enhanced chemiluminescence reagents (Animal Genetics Inc., Suwon, Kyonggi-do, Korea).

RNA isolation and real-time PCR. Cells were treated with various doses of $\mathrm{BC}$ for 4 days and total RNA extraction was performed on each sample using TRIzol reagent (Invitrogen). cDNA of $1 \mu \mathrm{g}$ total RNA was synthesized by reverse transcription with RevertAid First Strand cDNA synthesis kit (Thermo Fisher Scientific, Waltham, MA, USA), and real-time PCR was performed with StepOnePlus $^{\mathrm{TM}}$ (Applied Biosystems, Foster City, CA, USA) to examine the relative DLK1 mRNA levels among the samples according to the manufacturer's instructions. PCR primers were as follows: human DLK1, 5'-CTG 
A

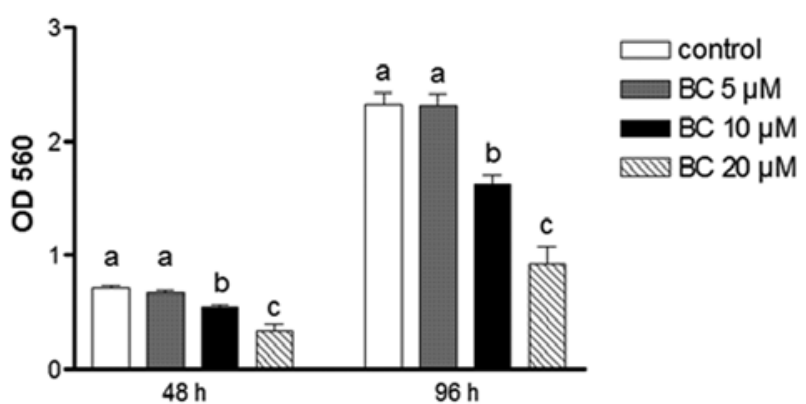

B

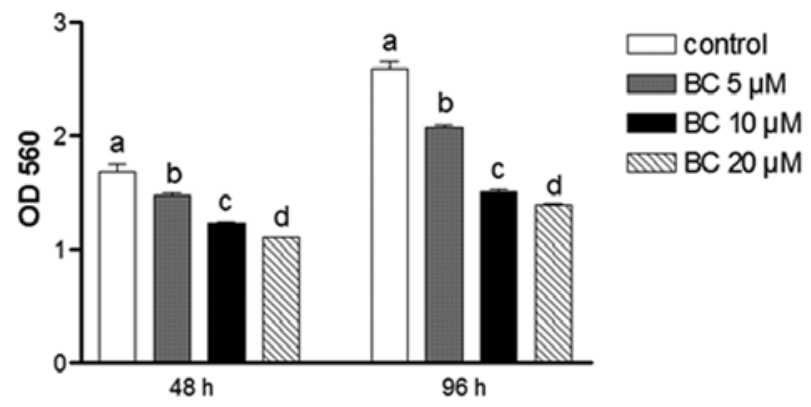

Figure 1. BC inhibits NB cell growth. Cell viability of (A) BE(2)C and (B) SY5Y cells was analyzed using MTT assay following treatment with various doses of $\mathrm{BC}$ for 48 or $96 \mathrm{~h}$. Values shown are the means \pm SEM derived from $>6$ replicate wells. The different letters at the top of the given bars of the histogram indicate that those values are significantly different from each other $(\mathrm{P}<0.05)$. BC, $\beta$-carotene.

AAGGTGTCCATGAAAGAG-3' (forward); 5'-GCTGAAGG TGGTCATGTCGAT-3' (reverse) and $\beta$-actin, 5'-ATTGGCA ATGAGCGGTTC-3' (forward); 5'-GGATGCCACAGGACT CCAT-3' (reverse). $\beta$-actin, a housekeeping gene, was used for equal RNA loading.

RNA interference. Small interfering RNA (siRNA) for human DLK1 (nM_003836) was purchased from Dharmacon (Lafayette, CO, USA), and the 5'-GCACCUGCGUGGAUGU GAUU-3' oligonucleotides were used to target nucleotides 578-596 of the DLK1 gene. Non-targeting siRNA (5'-UAACA AUGAGAGCACGGCUUU-3') was used as a transfection control. siRNA transfection was performed using DharmaFECT (Dharmacon) according to the manufacturer's instructions.

Statistical analysis. All results are expressed as the means \pm SEM. Comparisons between groups were performed using one-way analysis of variance (ANOVA) followed by the Newman-Keuls post-hoc test. All experiments were repeated at least three times to generate statistically relevant data. Data were considered significant when the P-value was $<0.05$.

\section{Results}

Effects of BC on NB cell growth. Cell growth of BE(2)C and SY5Y cells following 48 or $96 \mathrm{~h}$ of treatment with BC was evaluated using the MTT assay. Treatment with BC significantly decreased the viability of both cell types when compared to the controls at both time-points (Fig. 1A and 1B). For example, the viability of the $\mathrm{BE}(2) \mathrm{C}$ cells was decreased by $60 \%$ after treatment with $20 \mu \mathrm{M}$ of BC for $96 \mathrm{~h}$, while the same treatment decreased the viability of SY5Y cells by $46 \%$. Furthermore, these inhibitory effects were not due to toxicity since cell viability for each treatment was found to be $>90 \%$ (data not shown). Compared to the N-type cells, SY5Y, the I-type cells, $\mathrm{BE}(2) \mathrm{C}$, are more aggressive and less differentiated. As a result, $\mathrm{BE}(2) \mathrm{C}$ cells exhibit more stem cell-like characteristics than SY5Y cells (2) and accordingly, were preferentially used in the following experiments to examine the effect of BC on NB cell stemness.

Effects of BC on cell differentiation. $\mathrm{BE}(2) \mathrm{C}$ cells are I-type NB cells that possess cancer cell stemness and undergo morphological and functional differentiation in vitro (20). To examine the effect of $\mathrm{BC}$ on $\mathrm{NB}$ cell differentiation, cells were treated with various concentrations of $\mathrm{BC}$. Following treatment, $\mathrm{BE}(2) \mathrm{C}$ cells underwent morphological differentiation in a dose-dependent manner manifested by an increase in neurite growth, which is a hallmark of neuronal differentiation (Fig. 2A and B). In these assays, RA was used as a positive control to induce neuronal differentiation. The observed increase in neuronal differentiation following treatment with $\mathrm{BC}$ was confirmed with an upregulation of $\beta$-tubulin III that was detected by western blotting (Fig. 2C). Neurite elongation and protein expression profiles determined following $\mathrm{BC}$ treatment were also very similar to those obtained following RA treatment.

$B C$-induced neuronal cell differentiation involves ERK phosphorylation. It has been reported that ERK1/2 (p42/p44) is phosphorylated during the differentiation of neuronal progenitor cells (8). Similarly, when phosphorylation of ERK1/2 was assessed following treatment with $\mathrm{BC}$, a marked increase was observed, whereas the expression of total ERK was unchanged (Fig. 3A). To examine whether MAPK pathways are involved in BC-induced NB cell differentiation, the MAPK-specific inhibitors, U0126 and PD98059, were used. In these studies, phosphorylation of ERK1/2 was completely blocked following treatment with U0126 and 10 or $20 \mu \mathrm{M} \mathrm{BC}$, while phosphorylation of ERK1/2 was only partially blocked by PD 98059 at both concentrations of BC (Fig. 3B). Consistent with these results, $\mathrm{BC}$-induced neurite elongation was also completely blocked by U0126 treatment, and only partially blocked by PD 98059 (Fig. 3C). In combination, these data clearly demonstrate that $\mathrm{BC}$ induces neuronal differentiation of $\mathrm{NB}$, and plays an important role in the MAPK phosphorylation that is involved in this process.

$B C$ suppresses the self-renewal characteristics of $N B$. One of the more notable characteristics of CSCs is the capacity for self-renewal. Clonogenic assay measures the ability to form a colony from a single cell and represent the self-renewal potential of CSCs. Folowing the treatment of NB cells with 10 or $20 \mu \mathrm{M} \mathrm{BC}$, clonogenic formation was significantly suppressed when compared to the controls (Fig. 4A and B). Another widely used assay to analyze the self-renewal capacity of CSCs is sphere formation assay, which assesses the ability to grow as non-adherent spheroids in a specific serum-free medium. The NB cells grown in serum-free medium were able to form 
A
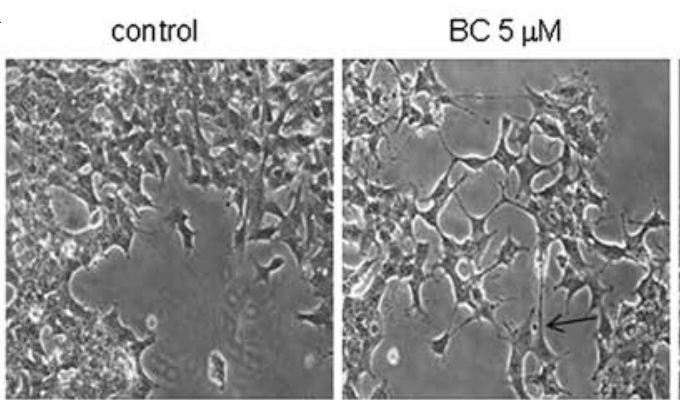

$\mathrm{BC} 10 \mu \mathrm{M}$

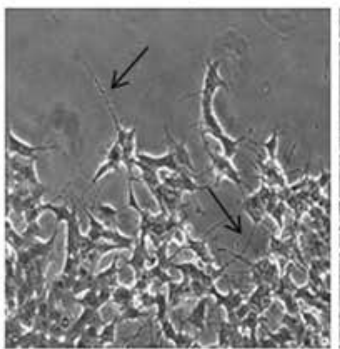

$\mathrm{BC} 20 \mu \mathrm{M}$

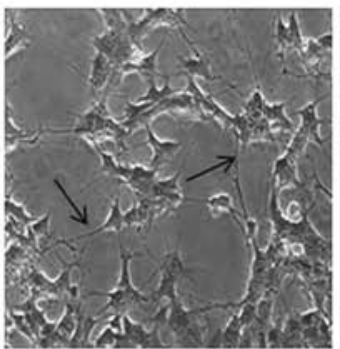

$\mathrm{RA} 1 \mu \mathrm{M}$

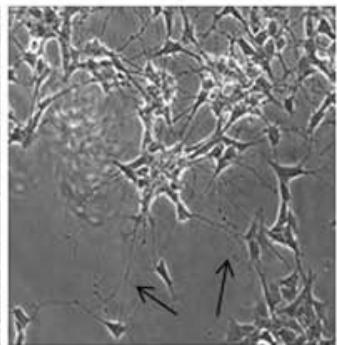

B

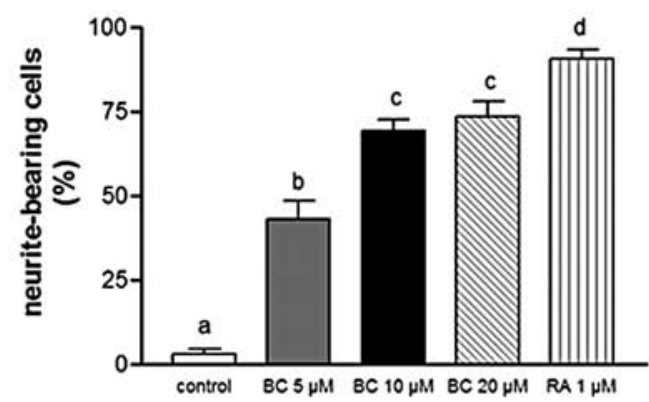

C

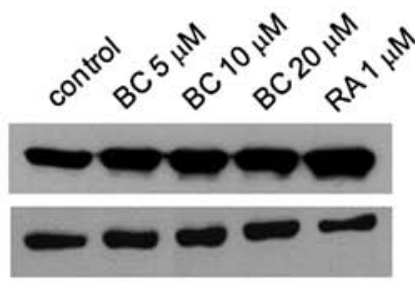

Figure 2. BC induces neuronal cell differentiation. (A) Phase contrast microscopy was used to examine the morphological features of differentiated BE(2)C cells following treatment with $\mathrm{BC}$ for 5 days. RA $(1 \mu \mathrm{M})$ was used as a positive control. Neuronal differentiation was evaluated based on the number of lengthened neurites (indicated with arrows) that were counted. Magnification, x100. (B) The percentage of neurite-bearing cells was calculated from samples plated in triplicate. At least three independent experiments were performed, and the means \pm SEM are presented. Different letters at the top of the given bars of the histogram indicate that those values are significantly different from each other $(\mathrm{P}<0.05)$. (C) Expression of neuron-specific $\beta$-tubulin III was analyzed by western blotting with detection of $\beta$-actin used as a loading control. BC, $\beta$-carotene; RA, all-trans-retinoic acid.
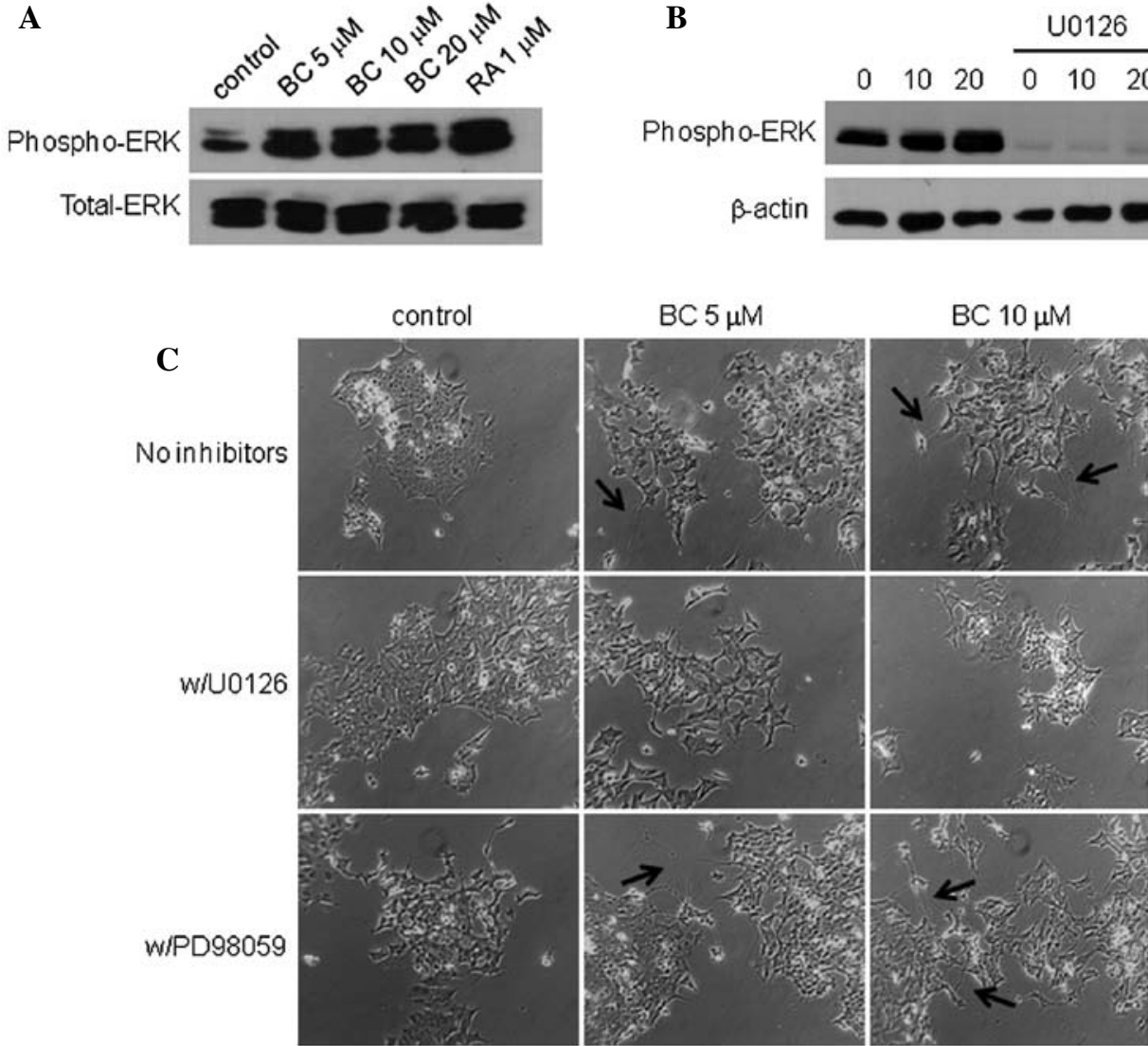

Figure 3. BC-induced neuronal differentiation involves ERK phosphorylation. BE(2)C cells were treated with BC for 5 days and (A) ERK phosphorylation was analyzed by western blotting with detection of total-ERK used as a loading control. (B and C) Cells were treated with various doses of BC, then with each MEK/ERK inhibitor (U0126 and PD98059) on the next day. After 3 days of incubation, immunoblots (B) and cell morphology (C) were analyzed. $\beta$-actin was used as a loading control, and arrows indicate lengthened neurites representative of neuronal differentiation. $\mathrm{BC}, \beta$-carotene. 
A

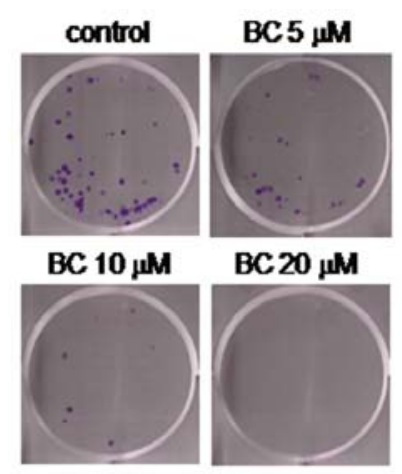

C

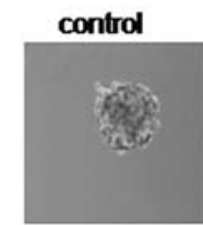

$\mathrm{BC} 10 \mu \mathrm{M}$

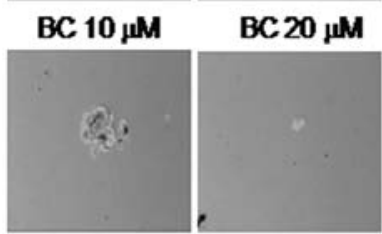

B

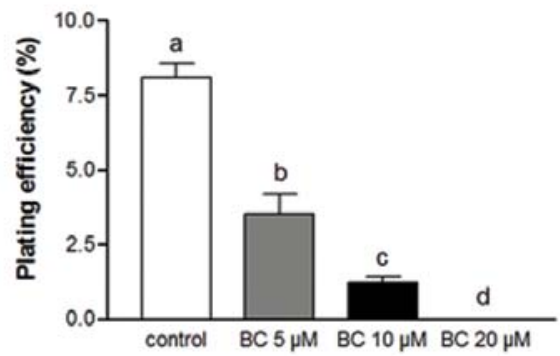

D

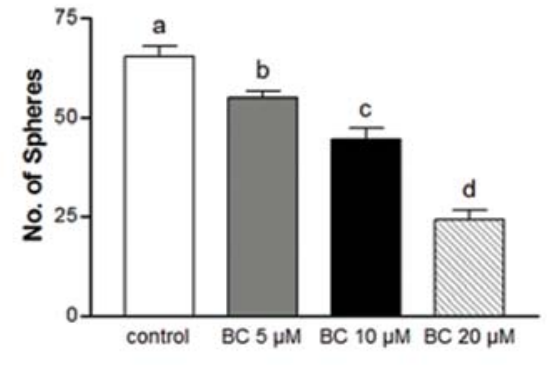

$\mathbf{E}$

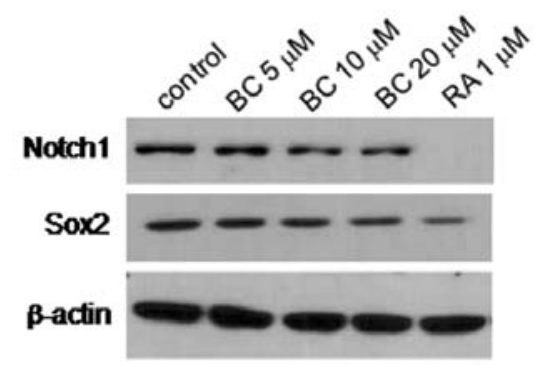

Figure 4. BC attenuates the self-renewal capacity of NB. (A) BE(2)C cells in 6-well plates were treated with $0,5,10$ or $20 \mu \mathrm{M}$ BC. After 7-10 days, colonies were stained and imaged. (B) The number of colonies with $\geq 50$ cells were counted, and plating efficiency $(\mathrm{PE})$ was calculated: $\mathrm{PE}=$ percentage of colonies per input \pm SEM; $(n=6, P<0.05$ vs. control). (C and D) $\mathrm{BE}(2) \mathrm{C}$ cells were also cultured in polyHEMA-coated tissue culture dishes and treated with various doses of BC in serum-free sphere media. After 6 days, sphere formation was imaged and the number of spheres was counted. Magnification, $\mathrm{x} 100$. Experiments were independently performed at least three times. Different letters at the top of the given bars of the histogram indicate that those values are significantly different from each other $(\mathrm{P}<0.05)$. (E) Representative immunoblot assay of Notch1 and Sox 2 expression in $\mathrm{BE}(2) \mathrm{C}$ cells following 4 days of treatment with various concentrations of BC or RA. BC, $\beta$-carotene; NB, neuroblastoma; RA, all-trans-retinoic acid.

a clonosphere and following treatment with $\mathrm{BC}$ exhibited a marked decrease in clonosphere formation compared to the controls $(\mathrm{P}<0.05)$, and this decrease was dose-dependent (Fig. 4C and D). To further verify the effect of $\mathrm{BC}$ on neuronal stem cell markers, BE(2)C cells were treated with BC for 4 days and the protein expression of several well-known stem cell markers, including Sox 2 and Notch1 was assessed. The expression of these stem cell markers was found to be downregulated (Fig. 4E). Taken together, these results suggest that $\mathrm{BC}$ is highly effective in decreasing the self-renewal capacity of NB cells, and in eliminating most NB stem cells.

BC downregulates DLK1 expression. DLK1 is recognized as a neuronal stem cell marker and has been hypothesized to play an important role in the growth of stem/progenitor cells and cancer cells (20). Here, in cells treated with BC, mRNA and protein levels of DLK1 were found to be downregulated (Fig. 5A and B). To determine whether a combination of $\mathrm{BC}$ treatment and knockdown of DLK1 inhibits cancer cell stemness, BE(2)C cells were transfected with siRNA oligonucleotides designed to target DLK1. Subsequently, endogenous expression of DLK1 was found to be completely abrogated
(Fig. 5C). Moreover, in the clonogenicity assay, treatment with BC or knockdown of DLK1 significantly inhibited colony formation (22 and 49\%, respectively, $\mathrm{P}<0.05$ ). Furthermore, the combination of BC treatment and DLK1 knockdown resulted in a $65 \%$ inhibition in clonogenicity compared to the control (Fig. 5D). Collectively, inhibition of DLK1 by siRNA was found to potentiate $\mathrm{BC}$-mediated inhibition of the self-renewal capacity of NB cells. Consequently, BC treatment with DLK1 knockdown may provide a more effective chemotherapeutic effect by regulating CSCs.

$B C$ potentiates the anticancer effect of cisplatin by inhibiting the self-renewal capacity of NB cells. CSCs resist conventional chemotherapeutic drugs and are likely to play an important role in cancer relapse. Therefore, it is important to develop cancer therapeutic agents targeting CSCs. Furthermore, most chemotherapeutic agents have undesirable side-effects. Therefore, various agents are administered in combination to increase their therapeutic efficacy while decreasing associated toxicity. Cell growth was examined using MTT assays to assess whether $\mathrm{BC}$ treatment in the presence of cisplatin has an additive effect on BE(2)C cells (Fig. 6A and B). For these assay, 
A

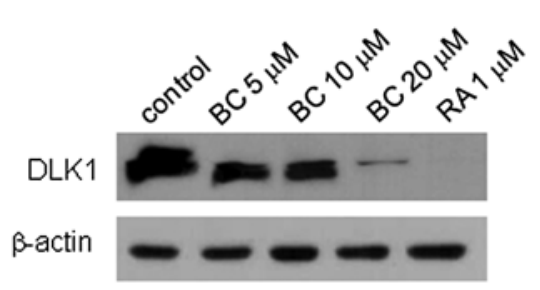

C

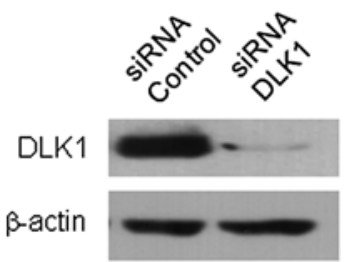

B

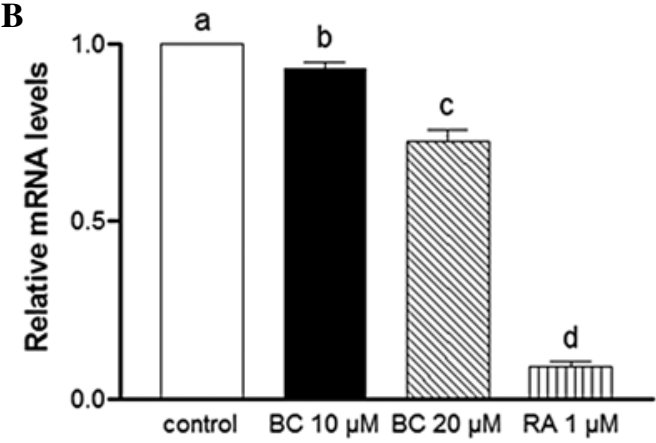

D

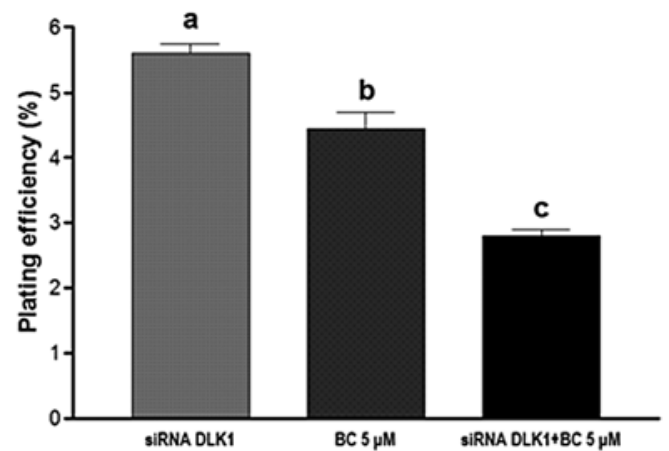

Figure 5. DLK1 expression is downregulated by BC. (A) Protein expression of DLK1 was assessed by western blotting 4 days after BE(2)C cells were treated with BC or RA. (B) Real-time PCR was performed to analyze levels of DLK1 mRNA following the treatment of BE(2)C cells with BC or RA for 3 days. $\beta$-actin was used as an internal control. Different letters at the top of the given bars of the histogram indicate that those values are significantly different from each other $(\mathrm{P}<0.05)$. (C) BE(2)C cells were transfected with DLK1-specific or non-targeting control siRNA. After $48 \mathrm{~h}$, cell lysates were prepared, and expression of DLK1 was analyzed by western blotting using $\beta$-actin as a loading control. (D) Clonogenic assays were performed to examine the self-renewal capacity of the cells described in Fig. 4. BC, $\beta$-carotene; RA, all-trans-retinoic acid

A

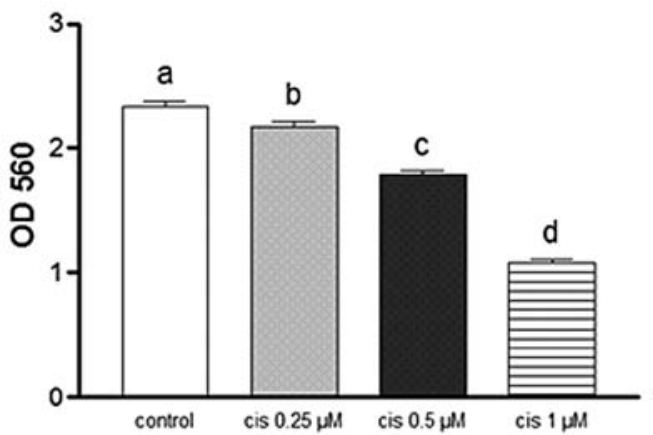

C

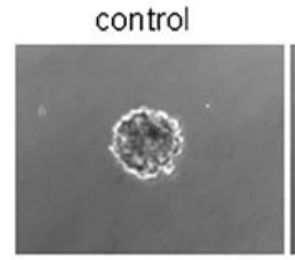

$\mathrm{BC} 10 \mu \mathrm{M}$

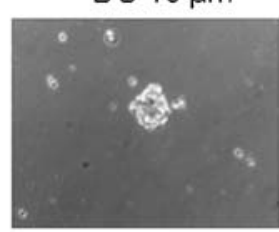

B

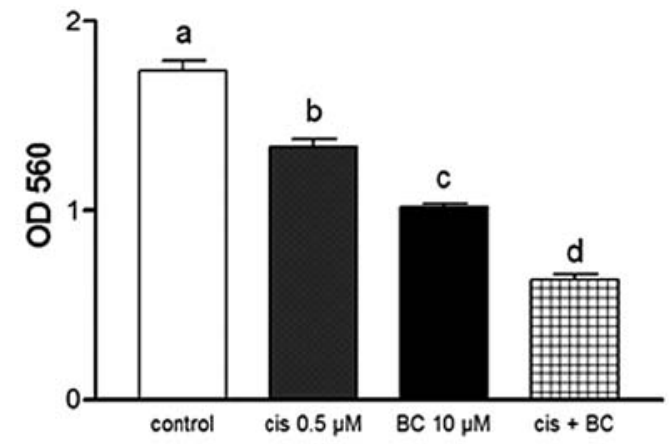

D

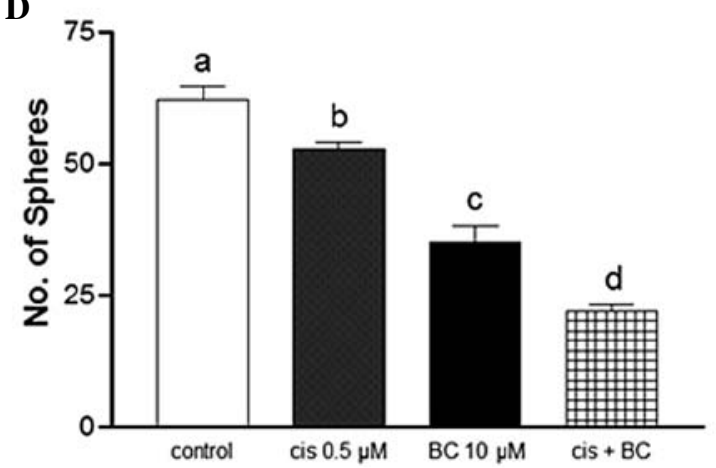

Figure 6. Combination treatment with BC and cisplatin decreases stem cell characteristics. (A) Cell growth inhibition by cisplatin was determined using MTT assays. $\mathrm{BE}(2) \mathrm{C}$ cells were treated with various doses of cisplatin for 4 days. (B) Cell growth inhibition in response to treatment with $\mathrm{BC}$ and cisplatin was analyzed. $\mathrm{BE}(2) \mathrm{C}$ cells in 6-well plates were treated with cisplatin $(0.5 \mu \mathrm{M}), \mathrm{BC}(10 \mu \mathrm{M})$ or both agents $(0.5 \mu \mathrm{M}$ cisplatin $+10 \mu \mathrm{M} \mathrm{BC})$. After 4 days, cell viability was analyzed using MTT assay. (C and D) Sphere formation assay was performed for BE(2)C cells treated with a combination of cisplatin and BC as described in Fig. 4. Spheres were imaged and counted. Magnification, x100. BC, $\beta$-carotene; cis, cisplatin. 
cells were treated with increasing doses of $\mathrm{BC}$ and cisplatin over 4 days. $\mathrm{IC}_{30}$ values calculated from the dose-response data obtained were $\sim 0.59 \mu \mathrm{M}$ for cisplatin (Fig. 6A) and $\sim 10.8 \mu \mathrm{M}$ for BC (Fig. 1A). To evaluate the antitumor effects of BC in combination with cisplatin, NB cells were treated with $0.5 \mu \mathrm{M}$ cisplatin, $10 \mu \mathrm{M} \mathrm{BC}$, or both. After 4 days, cell growth was analyzed using MTT assay. Whereas treatment with BC or cisplatin alone significantly decreased cell viability, the combination of $\mathrm{BC}$ with cisplatin further suppressed NB cell growth (Fig. 6B). Furthermore, to evaluate the effects of BC and cisplatin on the self-renewal characteristics of CSCs, a sphere formation assay was also conducted. The combination treatment had an additive effect on reducing sphere formation although either $\mathrm{BC}$ or cisplatin showed inhibition of sphere formation (Fig. 6C and D). These results suggest that $\mathrm{BC}$ has the potential to suppress the growth of CSCs, and the combination of $\mathrm{BC}$ with conventional chemotherapeutic agents could represent a more effective regimen for the treatment of this deadly childhood cancer.

\section{Discussion}

Neuroblastoma is the most common malignant tumor of the neural crest and arises within the sympathetic nervous system. In addition, the non-differentiated stem cell phenotype associated with NB has been identified as a potential independent risk factor for NB malignancy. Therefore, NB cells represent a model system applicable to the study of cancer stem cell-like cells. In the present study, treatment with BC was observed to induce the differentiation of NB cells and to decrease the selfrenewal characteristics of CSCs. Therefore, BC may represent a novel therapeutic strategy for the targeting of CSCs associated with $\mathrm{NB}$, thereby preventing recurrence and metastasis.

RA is a widely used chemotherapeutic drug that induces cell differentiation. Similarly, carotenoids have also been shown to induce cell differentiation $(23,24)$. For example, in a study by Gross et al (23), both BC and lutein were shown to induce the differentiation of HL-60 cells. $\beta$-cryptoxanthin, one of the major carotenoids present in blood, has also been shown to induce the morphological differentiation of mouse Neuro2a cells, in vitro (24). Prasad et al (25) also successfully differentiated the murine NB cell line, NBA2, by treating cells with $\mathrm{BC}$ for 3 days, followed by irradiation with X-rays at a dose of $20 \mathrm{~Gy}$ and higher. However, evidence regarding the differentiation signaling induced by $\mathrm{BC}$, and the efficacy of $\mathrm{BC}$ in regard to $\mathrm{CSC}$, remains unclear. In this present study, phosphorylation of ERK (p42/p44) was observed to markedly increase following treatment with BC. Moreover, the use of specific ERK inhibitors, PD98059 and U0126, confirmed the involvement of the ERK/MEK1/2 pathway in $\mathrm{BC}$-induced neuronal differentiation. It has previously been proposed that U0126 directly inhibits MEK1/2 activity, whereas PD98059 is an inefficient inhibitor of MEK2 (26). The differential effects of these inhibitors on osteogenesis and adipogenesis have also been characterized (27). Similarly, in a previous study of RA-induced neurite growth, growth was completely blocked by U0126, yet only partially blocked by PD98059 (28). Collectively, these data suggest that $\mathrm{BC}$-induced neuronal differentiation requires the inhibition of both MEK1 and MEK2. Furthermore, BC-induced neuronal differentiation appears to be partly mediated via a pathway also used for RA-induced neuronal differentiation. Therefore, the mechanistic details of the regulation of $\mathrm{BC}$ on ERK phosphorylation, as well as on the phosphorylation of other MAPKs require further analysis. However, our results suggest that ERK is an important mediator of BC-mediated neuronal differentiation.

In the present study, $\mathrm{BC}$ induced the neuronal differentiation of human NB cells, and several mechanisms could account for this observation. Folowing are examples proposed for NB differentiation induced by $\mathrm{BC}$ : i) $\mathrm{BC}$ could be converted to RA since $\mathrm{BC}$ is a precursor of RA and then cleaved via a central cleavage pathway (29); ii) differentiation may be induced by nuclear receptor-binding activities, subsequently leading to the induction of several transcription factors and signaling pathways involved in differentiation; or iii) RA signaling could be promoted by metabolites of BC. Further studies are needed to investigate these possibilities, as well as to determine the contributions of $\mathrm{BC}$ to the differentiation of CSCs and RA precursor function.

The self-renewal capacity of CSCs is associated with their tumorigenic potential (4). For example, the ability of CSCs to form colonies as well as anchorage-independent spheres is directly proportional to the proportion of self-renewing cells present. Correspondingly, an evaluation of these two characteristics can determine the effect of a particular treatment on CSCs. In the present study, NB cells were observed to form colonies and spheroids in the absence of $\mathrm{BC}$, yet in the present of $\mathrm{BC}$, the formation of colonies and spheres was reduced. A decrease in the levels of stem cell markers, DLK1, Notch1 and Sox 2 was also noted in BC-treated cells.

DLK1 is a transmembrane protein that is expressed extensively in immature cells with regenerative potential $(30,31)$. It has previously been reported that various tumors express higher levels of DLK1 $(32,33)$, and exogenous expression of DLK1 increases the self-renewal capacity of NB cells. In contrast, knockdown of $D L K 1$ has been shown to enhance neuronal differentiation (20). In the present study, the combination of BC treatment with knockdown of $D L K 1$ resulted in a significantly higher inhibition of colony formation than treatment with either agent alone. Since DLK1 is involved in MAPK signaling, it is hypothesized that BC and DLK1 also have roles in the same ERK pathway. Taken together, these results suggest that BC inhibits the self-renewal capacity of CSCs. Moreover, poorly differentiated tumors have recently been shown to have similar gene expression profiles as normal embryonic stem cells or progenitor cells $(34,35)$. However, studies are ongoing to further elucidate the mechanisms by which stem cell genes regulate tumor progression and metastasis, as well as the role of nutrients and tumor microenvironments.

Although initially responsive to cytotoxic treatment, most tumors acquire a multidrug-resistant (MDR) phenotype and relapse. CSCs are likely to play a major role in chemotherapy resistance and cancer relapse. Therefore, it is important to develop therapies that bypass the drug resistance of CSCs. Currently, the most promising strategy for the treatment of cancer is the administration of two or more drugs with different mechanisms of action to produce synergistic, or at least additive anticancer effects. Cisplatin was the first platinum-derived drug produced, and is still used as a first-line 
therapeutic agent against several types of cancers, including NB (36). While the chemotherapeutic effects of cisplatin are based on the formation of an adduct with DNA and proteins, as well as the disruption of several signaling pathways $(37,38)$, the administration of cisplatin is also associated with serious side-effects, including nephrotoxicity and peripheral neuropathy due to immune suppression (39). Due to these toxicities and undesirable side-effects, equally effective and safer treatments are needed. It has been reported that a combination treatment of minocycline with BC or 13-cis-retinoic acid, along with cisplatin, can result in decreased cytotoxicity (40). Furthermore, a mixture of three or four vitamins containing $\mathrm{BC}$ showed the potential to enhance the growth-inhibitory effects of cisplatin on melanoma cells (41). In the present study, we demonstrated for the first time that $\mathrm{BC}$ can potentiate the cytotoxic effects of cisplatin against NB CSCs. In detail, BC increased the self-renewal activity of NB cells in vitro and mediated a reduction in sphere formation. In the latter case, we hypothesize that this role for $\mathrm{BC}$ contributed to the resensitization of CSCs to the chemotherapeutic agent, cisplatin. Further study of the molecular mechanisms of $\mathrm{BC}$ in relation to the effects of chemotherapeutic agents are needed to confirm this hypothesis, and are warranted to develop more potent and less toxic combination therapies. However, our results are based on in vitro cell culture rather than in vivo. Therefore, further in vivo studies using animals should be performed to support and confirm the present results.

In conclusion, the present study revealed that $\mathrm{BC}$ exhibits excellent anti-CSC qualities, and can induce neuronal cell differentiation via phosphorylation of MAPK and downregulation of DLK1. For example, treatment with BC decreased the expression of stem cell markers and suppressed the selfrenewal characteristics of the NB cells assayed, including their colony and spheroid formation potential. Furthermore, our results suggest that $\mathrm{BC}$ may represent a potent medical adjunct for the treatment of NB in combination with cisplatin. However, additional studies are warranted to elucidate the mechanisms of action of $\mathrm{BC}$ in regard to both neuronal cell differentiation and inhibition of CSCs, thereby facilitating the application of $\mathrm{BC}$ as a therapeutic treatment.

\section{Acknowledgements}

This research was supported by the National Research Foundation of Korea (project no. 2011-0008169). The authors sincerely appreciate this support.

\section{References}

1. Brodeur GM: Neuroblastoma: biological insights into a clinical enigma. Nat Rev Cancer 3: 203-216, 2003.

2. Ciccarone V, Spengler BA, Meyers MB, Biedler JL and Ross RA: Phenotypic diversification in human neuroblastoma cells: expression of distinct neural crest lineages. Cancer Res 49: 219-225, 1989.

3. Reya T, Morrison SJ, Clarke MF and Weissman IL: Stem cells, cancer, and cancer stem cells. Nature 414: 105-111, 2001.

4. Clarke MF, Dick JE, Dirks PB, et al: Cancer stem cells - perspectives on current status and future directions: AACR Workshop on Cancer Stem cells. Cancer Res 66: 9339-9344, 2006.

5. Smas CM and Sul HS: Pref-1, a protein containing EGF-like repeats, inhibits adipocyte differentiation. Cell 73: 725-734, 1993.
6. Moore KA, Pytowski B, Witte L, Hicklin D and Lemischka IR: Hematopoietic activity of a stromal cell transmembrane protein containing epidermal growth factor-like repeat motifs. Proc Natl Acad Sci USA 94: 4011-4016, 1997.

7. Hunter T: Protein kinases and phosphatases: the yin and yang of protein phosphorylation and signaling. Cell 80: 225-236, 1995.

8. Berwick DC, Calissano M, Corness JD, Cook SJ and Latchman DS: Regulation of Brn-3a N-terminal transcriptional activity by MEK1/2-ERK1/2 signalling in neural differentiation. Brain Res 1256: 8-18, 2009.

9. Ruiz-Hidalgo MJ, Gubina E, Tull L, Baladron V and Laborda J: dlk modulates mitogen-activated protein kinase signaling to allow or prevent differentiation. Exp Cell Res 274: 178-188, 2002.

10. Burdon T, Smith A and Savatier P: Signalling, cell cycle and pluripotency in embryonic stem cells. Trends Cell Biol 12: 432-438, 2002.

11. Mayne ST: Beta-carotene, carotenoids, and disease prevention in humans. FASEB J 10: 690-701, 1996.

12. Comstock GW, Alberg AJ, Huang HY, et al: The risk of developing lung cancer associated with antioxidants in the blood: ascorbic acids, carotenoids, $\alpha$-tocopherol, selenium, and total peroxyl radical absorbing capacity. Am J Epidemiol 168: 831-840, 2008.

13. Liu C, Russell RM and Wang XD: Low dose $\beta$-carotene supplementation of ferrets attenuates smoke-induced lung phosphorylation of JNK, p38 MAPK, and p53 proteins. J Nutr 134: 2705-2710, 2004.

14. Burton GW and Ingold KU: beta-Carotene: an unusual type of lipid antioxidant. Science 224: 569-573, 1984.

15. Tighe AP and Gudas LJ: Retinoic acid inhibits leukemia inhibitory factor signaling pathways in mouse embryonic stem cells. J Cell Physiol 198: 223-229, 2004.

16. Fenaux P, Chevret S, Guerci A, et al: Long-term follow-up confirms the benefit of all-trans retinoic acid in acute promyelocytic leukemia. European APL group. Leukemia 14: 1371-1377, 2000.

17. Kakarala M, Brenner DE, Korkaya H, et al: Targeting breast stem cells with the cancer preventive compounds curcumin and piperine. Breast Cancer Res Treat 122: 777-785, 2010.

18. Corrao S, Scaglione R, Arnone S and Licata G: Left ventricular diastolic filling alterations in subjects with mitral valve prolapse: a Doppler echocardiographic study. Eur Heart J 14: 369-372, 1993.

19. Denizot F and Lang R: Rapid colorimetric assay for cell growth and survival. Modifications to the tetrazolium dye procedure giving improved sensitivity and reliability. J Immunol Methods 89: 271-277, 1986.

20. Kim Y, Lin Q, Zelterman D and Yun Z: Hypoxia-regulated delta-like 1 homologue enhances cancer cell stemness and tumorigenicity. Cancer Res 69: 9271-9280, 2009.

21. Folkman J and Moscona A: Role of cell shape in growth control. Nature 273: 345-349, 1978.

22. Lin Q, Lee YJ and Yun Z: Differentiation arrest by hypoxia. J Biol Chem 281: 30678-30683, 2006.

23. Gross MD, Bishop TD, Belcher JD and Jacobs DR Jr: Induction of HL-60 cell differentiation by carotenoids. Nutr Cancer 27: 169-173, 1997.

24. Noguchi S, Sumida T, Ogawa H, Tada M and Takahata K: Effects of oxygenated carotenoid beta-cryptoxanthin on morphological differentiation and apoptosis in Neuro2a neuroblastoma cells. Biosci Biotechnol Biochem 67: 2467-2469, 2003.

25. Prasad KN, Carvalho E, Kentroti S, et al: Production of terminally differentiated neuroblastoma cells in culture. Restor Neurol Neurosci 7: 13-19, 1994.

26. Favata MF, Horiuchi KY, Manos EJ, et al: Identification of a novel inhibitor of mitogen-activated protein kinase kinase. J Biol Chem 273: 18623-18632, 1998.

27. Dang ZC and Lowik CW: Differential effects of PD98059 and U0126 on osteogenesis and adipogenesis. J Cell Biochem 92: $525-533,2004$.

28. Kim Y: Effect of retinoic acid and delta-like 1 homologue (DLK1) on differentiation in neuroblastoma. Nutr Res Pract 4: 276-282, 2010.

29. Wang XD and Krinsky NI: The bioconversion of beta-carotene into retinoids. Subcell Biochem 30: 159-180, 1998.

30. Floridon $\mathrm{C}$, Jensen $\mathrm{CH}$, Thorsen $\mathrm{P}$, et al: Does fetal antigen 1 (FA1) identify cells with regenerative, endocrine and neuroendocrine potentials? A study of FA1 in embryonic, fetal, and placental tissue and in maternal circulation. Differentiation 66: 49-59, 2000. 
31. Tornehave D, Jensen CH, Teisner B and Larsson LI: FA1 immunoreactivity in endocrine tumours and during development of the human fetal pancreas; negative correlation with glucagon expression. Histochem Cell Biol 106: 535-542, 1996.

32. Sakajiri S, O'Kelly J, Yin D, et al: Dlk1 in normal and abnormal hematopoiesis. Leukemia 19: 1404-1410, 2005.

33. Yin D, Xie D, Sakajiri S, et al: DLK1: increased expression in gliomas and associated with oncogenic activities. Oncogene 25: 1852-1861, 2006.

34. Ben-Porath I, Thomson MW, Carey VJ, et al: An embryonic stem cell-like gene expression signature in poorly differentiated aggressive human tumors. Nat Genet 40: 499-507, 2008.

35. Phillips HS, Kharbanda S, Chen R, et al: Molecular subclasses of high-grade glioma predict prognosis, delineate a pattern of disease progression, and resemble stages in neurogenesis. Cancer Cell 9: 157-173, 2006.

36. Quasthoff S and Hartung HP: Chemotherapy-induced peripheral neuropathy. J Neurol 249: 9-17, 2002.

37. Huang H, Zhu L, Reid BR, Drobny GP and Hopkins PB: Solution structure of a cisplatin-induced DNA interstrand cross-link. Science 270: 1842-1845, 1995.
38. Donzelli E, Carfi M, Miloso M, et al: Neurotoxicity of platinum compounds: comparison of the effects of cisplatin and oxaliplatin on the human neuroblastoma cell line SH-SY5Y. J Neurooncol 67: 65-73, 2004.

39. Bruce AC: Cancer: Principles and practice of oncology. In: Anticancer Drugs. DeVita VT, Lawrence TS and Rosenberg SA (eds). JB. Lippincot Company, Washington, pp325-340, 1993.

40. Teicher BA, Schwartz JL, Holden SA, Ara G and Northey D: In vivo modulation of several anticancer agents by beta-carotene. Cancer Chemother Pharmacol 34: 235-241, 1994.

41. Prasad KN, Hernandez C, Edwards-Prasad J, Nelson J, Borus T and Robinson WA: Modification of the effect of tamoxifen, cisplatin, DTIC, and interferon- $\alpha 2 \mathrm{~b}$ on human melanoma cells in culture by a mixture of vitamins. Nutr Cancer 22: 233-245, 1994. 